\title{
Simulation of Superfinished Surface Formation
}

\author{
Augustín Görög ${ }^{1 *}$ \\ 1 Slovak University of Technology in Bratislava, Faculty of Materials Science and Technology in Trnava, J. Bottu \\ 25, 91724 Trnava, Slovak Republic \\ * Corresponding author's email: augustin.gorog@stuba.sk
}

\begin{abstract}
The article deals with simulating superfinishing of single-oscillating grooves. A model of the superfinishing tool was created according to the input information about the tool and the superfinishing process. Using this tool, a simulation of the cutting process was performed. The input parameters for the simulation were the cutting conditions, which determined the paths of individual grains of tools (process kinematics). The simulation is realized by gradual removal of the workpiece material by individual grains of the tool. The result of the simulation is the profile of the superfinished surface at an evaluation length of $1.25 \mathrm{~mm}$. It is possible to determine the surface roughness parameters from the profile. Creating and displaying multiple profiles side by side produces a graphical model of the superfinished surface. The simulation can be used for numerical simulation experiments, where both material and energy are saved. The article presents one such experiment together with the results - the surface roughness was influenced by individual factors. The simulation results (surface roughness parameters and surface appearance) were compared with the real superfinished surface on the outer ring of the bearing. This surface was manufactured under mass production conditions. The comparison confirmed that a simulation is a suitable tool for research of abrasive machining methods. At the end of the article, there is a discussion about a possible improvement of the simulation - considering 3D grains of the tool.
\end{abstract}

Keywords: simulation, model, surface roughness, superfinishing.

\section{INTRODUCTION}

The finishing machining methods of the metallic surfaces were conceived to be used specially to diminish the negative influences on the working accuracy and on the structural modification of materials which appear at the previous roughing and semifinishing operations, made by the temperature which arises at tool-part contact, by the destruction of the metal crystalline structure in the external layers and by large stress in the cutting zone [1].

According to the [2], superfinishing is the final stage in the manufacture of high-precision parts that take the form of solids of revolution. The main goal of superfinishing is the increase the precision of the cross-sectional profile of the part. It is assessed in terms of noncircularity-in particular, oval distortion, faceting, and undulation of the surface. Production experience shows that faceting and undulation may be effectively reduced in most cases, whereas it is much more difficult to reduce oval distortion.

The superfinishing process consists of three phases:

a) the cutting phase, which is characterized by a high material removal rate due to sharp cutting edges;

b) the transition phase, which is characterized by a decrease in the material removal rate due to dulling and loading of the stone; and

c) the finishing phase in which dulling and loading results in only slight or no material removal. In the subsequent superfinishing process, the loaded stone contacts the rough surface of the next workpiece to initiate self-dressing of the stone - providing sharp cutting edges - and the three phases described are repeated [3]. 
This article is focused on single-oscillating superfinishing of cylindrical workpieces, the movements of which consist of:

- oscillations of the superfinishing tool in the direction of the workpiece axis,

- tool pressure on the workpiece,

- rotating the workpiece around its axis.

However, superfinishing with more complex kinematics (multi-oscillating, centreless, ultrasonic, ...) is also commonly used. Zakharov in $[4,5]$ presented the theoretical principles for setting centreless superfinishing machines, which are derived by optimizing geometry, kinematic parameters and forces. Modelling and simulation of surface topography are functionally important. Owing to surface topography modelling, costs and time of experimental investigations can be substantially reduced. If the tribological properties of machine elements are accurately modelled, the optimal surface topographies used under particular tribological conditions can be generated, which may reduce the time and costs of experimental research [6].

Due to the great demands regarding the surface roughness which has a great influence on the service life and reliability of parts and the studies of the technical and economical performances of finishing processes were performed to optimize the process parameters and construction parameters of the abrasive tools. Other parameters that have a great influence on the surface roughness obtained through superfinishing are the dimensional and geometrical accuracy conditions at previous operations [1]. The modelling of machining by superfinishing according with the influences of various factors on the surface finish was performed in many articles.

In [7], the authors have proposed a new theoretical model of superfinishing process characterized by a system of a semifluid between abrasive tool and workpiece. This new theoretical model explains that the cutting process stopped itself by increasing the bearing surface and by filling the valley heights with microchips particles, abrasive particles and binder particles. Mathematical modelling was performed based on a spherical model of abrasive grain and similarity with broaching process. The authors [8] presented the basic ideas of modelling with finite elements of the elastic deformations at superfinishing process of external surfaces. The program they proposed to use for the superfinishing process can provide solutions to the issues related to elastic deformations and their effects on the accuracy of components at the design stage of the process. Jiang and Ge [9] presented the simulation method to form the topography on the condition of various combinations of applied loads, rotational speeds, reciprocating frequencies, and amplitude of superfinishing stones, the effect of every parameter on the topography was clarified. A numerical procedure for randomly generating any general three dimensional surface roughness with prescribed statistical properties was presented by [10]. Through the use of linear transformations on random matrices, this procedure was capable of generating Gaussian or non-Gaussian rough surfaces with any given surface autocorrelation function. The method developed by [10] was improved by Liao et al. [11] by transforming the solution of the system of non-linear equations to the non-linear least-squares problem. The NCGM method was applied to solve this problem. Moreover, FFT was used to improve the computational efficiency. In [12] a new technique for the synthesis of three-dimensional surfaces was developed. The method was based on the application of the discrete and continuous twodimensional Fourier transformations associated with the theory of discrete and continuous-time series models. This new technique facilitated the synthesis of three-dimensional surfaces with predetermined properties in any number of desired directions. The authors [13] compared the results obtained in simulation for the specific case of bearing rings ball track superfinishing with real superfinishing results. A good agreement of wear volumes was obtained and the simulation appeared effective. Arrazola [14] summarized the basic modelling structure, where three main parts can be observed: input parameters, models and output parameters. This structure for cutting processes is presented in Figure 1.

After modelling the surface topography, it is necessary to evaluate to surface characteristics. Two main reasons exist for the measurement of surface topography: quality control and (perhaps the more important one) prediction of the surface functional properties. Surface roughness is derived from the surface texture by retaining the short-wave component. Similarly, the waviness and roughness of $2 \mathrm{D}$ profiles exist. A primary profile corresponds to the surface texture. The analysis of the surface topography is difficult. Various parameters and functions are used to specify the roughness. [15] The authors [16] presented 


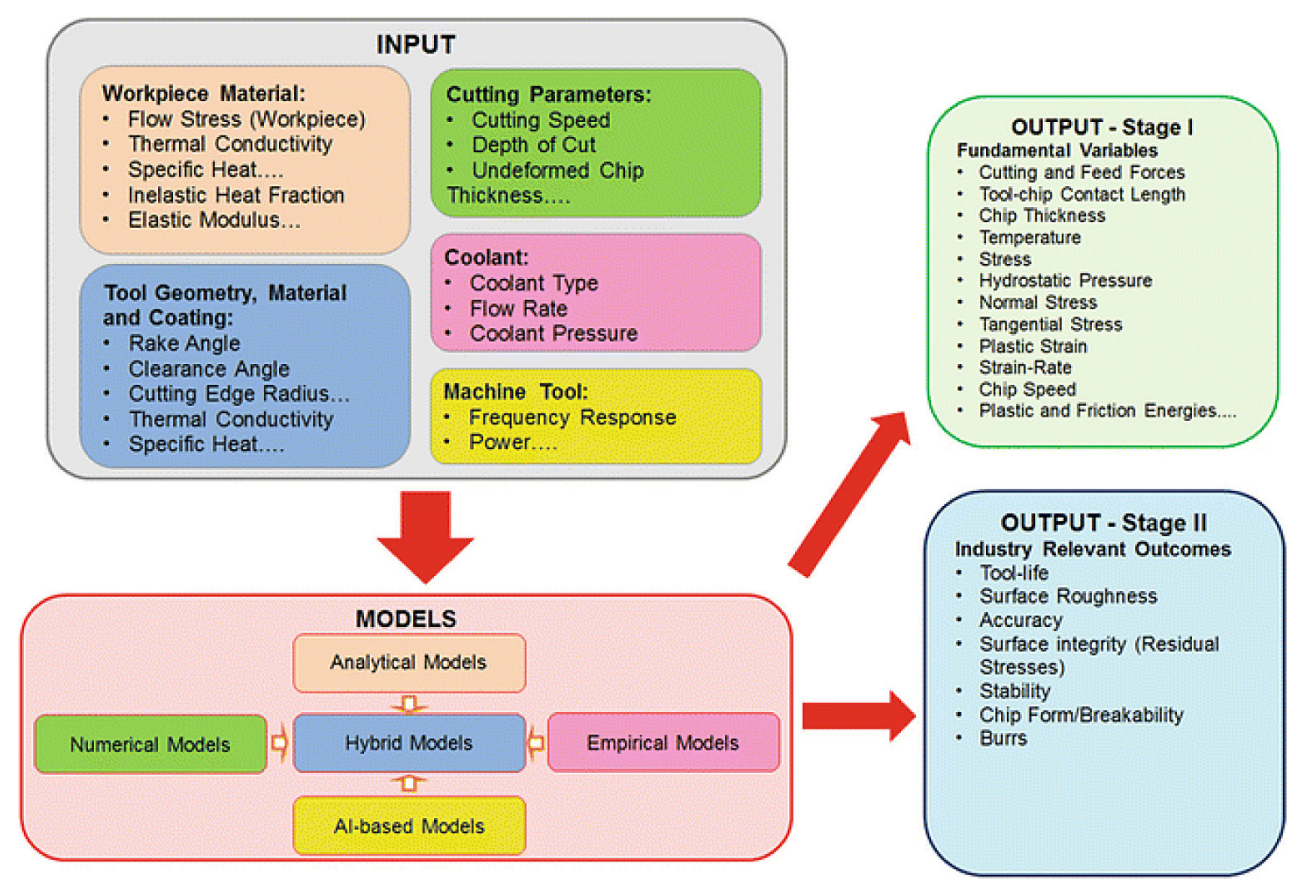

Fig. 1. Basic scheme for modelling of machining processes [14]

a possibility of surface roughness mathematical modelling in superfinishing on the process parameters and cutting fluid properties influence according to with the friction coefficient between abrasive grains and the workpiece surface. In [17], the Sugeno neuro-fuzzy model was derived for the superfinishing process to optimize the force and the speed parameters for reaching minimal average roughness $R a$. The Sugeno model made it possible to reduce friction, wear, energy consumption and service and maintenance costs.

This article deals with the simulation of single-oscillating grooves superfinishing and with creating the mathematical model of the superfinishing surface.

\section{SUPERFINISHING TOOL MODEL}

The roughness profile of the superfinished surface is obtained by simulating the removal of material from the workpiece. In order to do this, one must first create a tool model. The superfinishing tool is a multi-wedge cutting tool with an undefined geometry of the cutting wedges. When creating a model, it is necessary to consider a lot of information. In order not to make the model too complex, it is necessary to differentiate between the important and less important factors. The basic characteristics of the proposed model of the superfinishing tool can be briefly summarized in the following points:

- The superfinishing tool has a prism shape. Its lower surface is bevelled by the value of the layer of material removed after one pass of the superfinishing tool (Fig. 2). This chamfer is created directly in the superfinishing process (tool wear).

- It is not necessary to model the entire superfinishing tool. The model of the tool will form only its so-called "Active layer". It contains

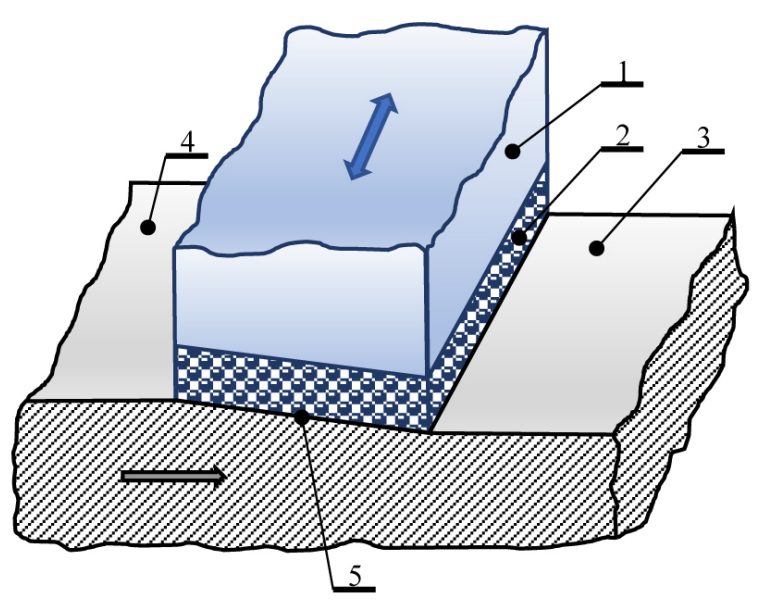

Fig. 2. Superfinishing tool model: 1 - superfinishing tool, 2 - active tool layer, 3 - machined workpiece surface, 4 - machined workpiece surface, 5 - workpiece cutting surface 
abrasive grains, which are directly involved in the removal of material in the form of chips. It is not necessary to model the active layer along the entire length of the tool. The modelled length is equal to the evaluated length of surface roughness $(\ln =1.25 \mathrm{~mm})$, increased on each side by half the value of twice the amplitude.

- The volume of the superfinishing tool consists of abrasive grains, binder and pores. The ratio of grain volume to total tool volume (grain volume + binder volume + pore volume) can be defined as a percentage. With a known mean grain diameter, it is possible to calculate the number of grains in the unit (and thus also in the modelled) volume of the tool. Assuming that the grains have the shape of a sphere, the same diameter, touch and are evenly distributed (like atoms in a cubic single lattice), they will fill $52.36 \%$ of the volume. In reality, however, these assumptions do not apply - the grains do not have the shape of a sphere, do not have the same diameter and do not touch each other (there is also a binder and pores between them).

- The individual grains are not regularly distributed in the real superfinishing tool. Their position is random. From a statistical point of view, it can be assumed that they are evenly distributed. Their position in the models is determined with the method of random numbers (Monte Carlo method).

- The shapes of the cutting edges of the individual grains in the superfinishing tool are different. For simplicity, 12 cutting edge shapes have been proposed, which have been randomly assigned to individual grains. No other geometry of the cutting wedge was considered here (angle of the forehead, angle of the back, ...).

- A rigid tool was considered when modelling the superfinishing tool.

- For simplicity, tool wear was not considered in superfinishing tool models.

\section{THE SIMULATION OF MATERIAL REMOVAL DURING SUPERFINISHING}

The numerical simulation of material removal during superfinishing consists of the mathematical connection of the model of the superfinishing tool with the kinematics of the superfinishing (mutual movement of the tool and the workpiece). In this case, single-oscillating recess superfinishing was simulated. The paths of the individual tool grains are formed by sinusoids (Fig. 3). The crossing angle $\beta$ after superfinishing depends on the maximum oscillation speed $v_{\text {omax }}$ and the workpiece speed $v_{W}$. The equation of oscillating motion is:

$$
y=\frac{A}{2} \sin \omega t
$$

where:

$$
\omega=2 \pi f
$$

The velocity of the oscillating motion is obtained by deriving the oscillation equation according to time:

$$
v_{o}=\frac{d y}{d t}=\frac{d\left(\frac{A}{2} \sin \omega t\right)}{d t}=\frac{A}{2} \omega \cos \omega t=\frac{A}{2} 2 \pi f \cos \omega t=A \pi f \cos \omega t
$$

The highest velocity of oscillating motion:

is when the condition is met:

$$
v_{\text {omax }}=A \pi f
$$

$$
\cos \omega t=1
$$

The circumferential velocity of the workpiece is:

$$
v_{w}=\pi d n
$$

The crossing angle of the tracks is:

$$
\operatorname{tg} \frac{\beta}{2}=\frac{v_{\text {omax }}}{v_{w}}=\frac{A \pi f}{\pi d n}=\frac{A f}{d n}
$$

where: $A$ - double amplitude, $f$ - oscillation frequency of the superfinishing tool, $d$ - workpiece diameter, $n$-workpiece rotation frequency, $\omega$ - angular velocity.

Figure 4 presents the simulation procedure at one sampling length of $l r=0.25 \mathrm{~mm}$. It is based on a surface with ideal (zero) roughness. The resulting profile only comprises traces of grains of the superfinishing 


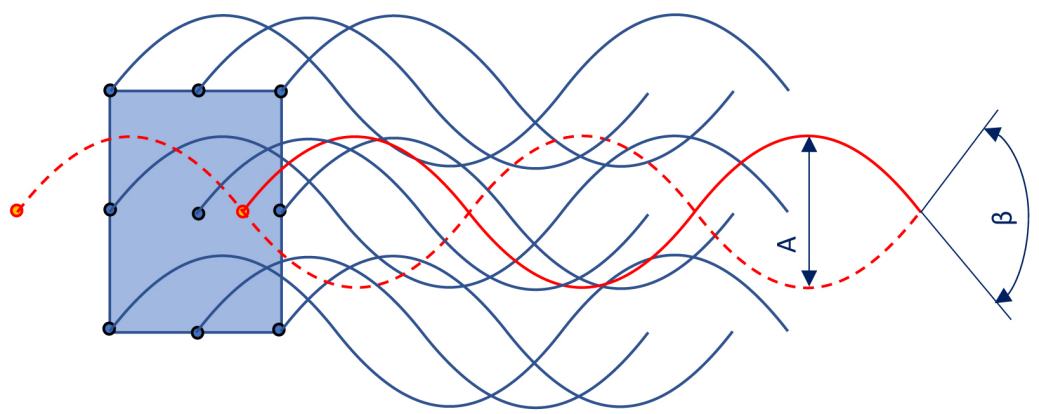

Fig. 3. Paths of individual grains of the superfinishing tool
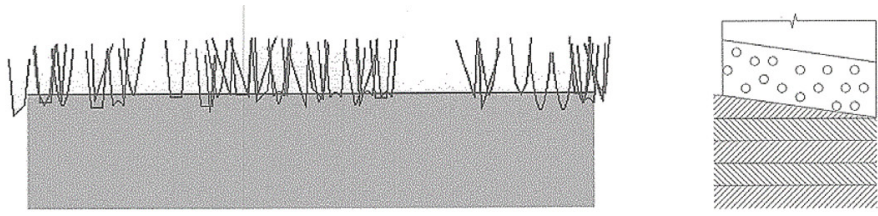

$a-1^{s t}$ the tool transition
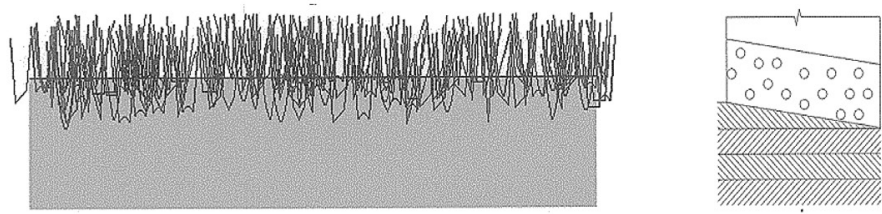

$b-2^{\text {nd }}$ the tool transition
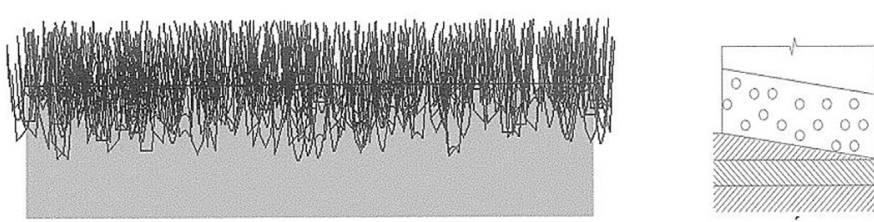

$c-3^{\text {rd }}$ the tool transition
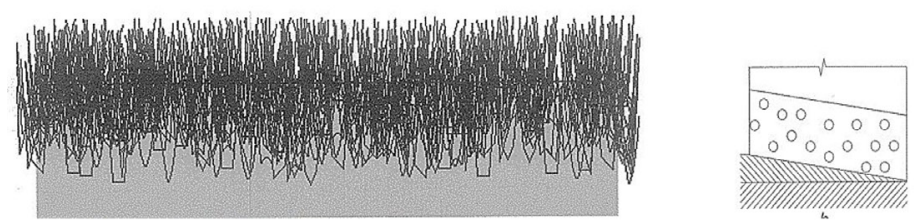

$d-4^{\text {th }}$ the tool transition
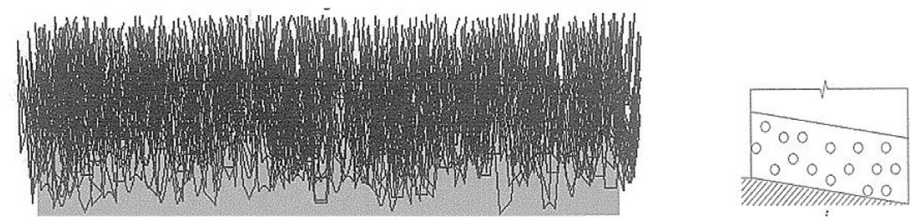

$e-5^{\text {th }}$ the tool transition

Fig. 4. Simulation of material removal during superfinishing - individual tool transitions

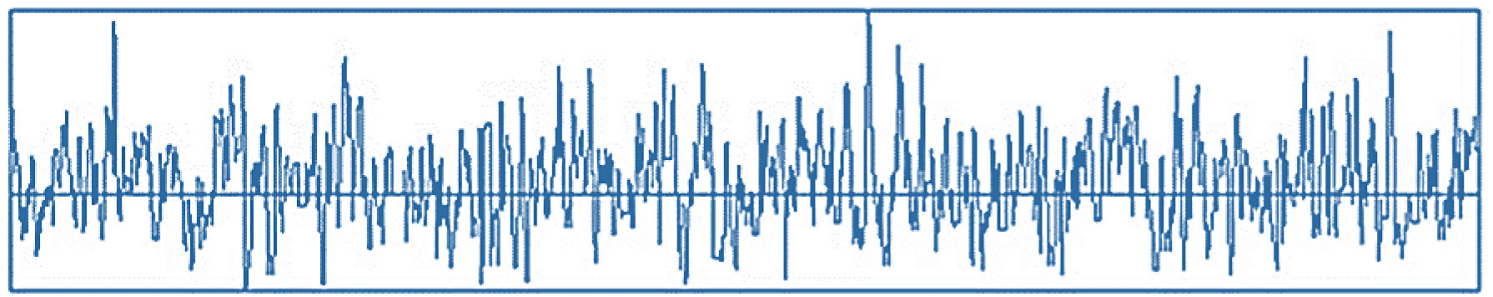

Fig. 5. Display of simulated roughness profile 
tool (the ground layer is removed). The input for the simulation software is the following data:

- double amplitude (e.g. $A=3 \mathrm{~mm}$ ),

- the oscillation frequency (e.g. $f=2000 \mathrm{~min}^{-1}$ ),

- the workpiece speed (e.g. $V_{W}=30 \mathrm{~m} \cdot \mathrm{min}^{-1}$ ),

- the phase shift (e.g. $\varphi=75^{\circ}$ ),

- the width of the superfinishing tool (e.g. $b=$ $10 \mathrm{~mm}$ ),

- the thickness of the material layer removed after one pass of the superfinishing tool (e.g. Uo $=0.25 \mu \mathrm{m}$ ),

- the grain size of the superfinishing tool (e.g. $Z$ $=22 \mu \mathrm{m}$ ),

- the percentage ratio of grain volume to tool volume (e.g. $p=52.36 \%$ ),

- the number of examined cuts on the workpiece (e.g. $n=350$ ),

- the distance between two adjacent cuts (e.g. $d$ $=0.002 \mathrm{~mm}$ ).

By simulation, we obtain a profile on the evaluated length $\ln =1.25 \mathrm{~mm}$. Following the usual rules, this evaluated length consists of five basic lengths $(\ln =5$. Ir). The profile obtained by the simulation can be displayed (Fig. 5). At the same time, it is possible to determine the surface roughness parameters from it $(R a=0.149 \mu \mathrm{m}, R q$ $=0.18 \mu \mathrm{m}, R z=1.05 \mu \mathrm{m}, R t=1.19 \mu \mathrm{m})$.

\section{GRAPHIC MODEL OF SUPERFINISHED SURFACE}

The simulation results can also be used to display a graphical model of the superfinished surface. During the simulation itself, it is possible to create the required number of surface roughness profiles. These profiles lie parallel to each other at a distance that must be entered with the input values. Then, it is possible to graphically display the side of the individual profile side by side. Thus the graphical model will be created. Individual profiles can be displayed in different ways. In Figure 6 the model of a simulated surface is presented, where profiles are shown using ten shades of grey. The points of the simulated profile lie on a horizontal line. The height of each profile point is displayed through the colour of the point. The higher the profile in the displayed location is, the paler the point is displayed. By gradually displaying the other profiles right next to each other (in the direction from the top of the picture below), a surface

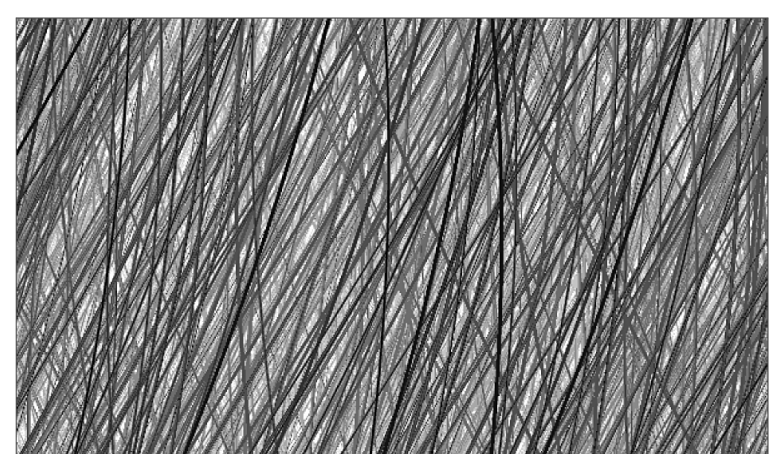

Fig. 6. Superfinished surface model

The surface size: $1.2 \times 0.7 \mathrm{~mm}(350$ cuts, distance between cuts $=0.002 \mathrm{~mm}$ )

model is created. This type of model displays a perpendicular view of the part surface (as a view of a real surface in a microscope).

\section{NUMERICAL SIMULATION EXPERIMENT}

The simulation was used for various numerical experiments. This saves material, energy and human labour. The experiment was carried out by the created simulation program. It aimed to determine the influence of production conditions of superfinishing on the surface roughness $R a$. The following three factors were selected from many factors affecting the surface roughness:

- the grain size of the superfinishing tool $Z \in<$ $15 \mu \mathrm{m} ; 32 \mu \mathrm{m}>$

- the angle of crossed traces $\beta \in<8,53^{\circ} ; 56,31^{\circ}>$,

o depends on the double amplitude $A$, the oscillation frequency $f$ and the workpiece speed $v_{W}$,

o for $\beta=8,53^{\circ}$ the parameters was: $A=2$ $\mathrm{mm}, f=1500 \mathrm{~min}^{-1}, V_{W}=40 \mathrm{~m} \cdot \mathrm{min}^{-1}$,

o for $\beta=56,31^{\circ}$ the parameters was: $A=$ $5 \mathrm{~mm}, f=3000 \mathrm{~min}^{-1}, v_{W}=20 \mathrm{~m} \cdot \mathrm{min}^{-1}$,

- the thickness of the removed layer of material after one pass of the superfinishing tool (depth of cut) $U_{o} \in<0.2 \mu \mathrm{m} ; 0.4 \mu \mathrm{m}>$.

After performing a numerical experiment and statistical analysis of the results, an equation for calculating the mean arithmetic deviation was found $R a[\mu \mathrm{m}]$ :

$$
R a=0.128\left(\frac{Z}{22}\right)^{1.032}\left(\frac{U o}{0.28}\right)^{0.405}
$$

The coefficient corresponding to the effect of the angle of crossed traces $\beta$ after superfinishing 
on the surface roughness $R a$ was statistically insignificant. This is because, in the model of the superfinishing tool, three-dimensional grains were not considered, but only with their two-dimensional image.

\section{THE COMPARISON OF SIMULATION RESULTS WITH REAL VALUES}

The results of the simulation correspond to the numerical values of the surface roughness $R a$ reported in the literature. The accuracy of the simulation was confirmed by comparing it with a real surface, which was produced under normal conditions of mass production. The surface roughness was measured on the superfinished surface of the outer ring of the bearing. The production parameters of this area were:

- the double amplitude of the tool vibration

$2.5 \mathrm{~mm}$

- the frequency of the tool vibration

$100 \mathrm{~min}^{-1}$

- the workpiece speed

- the width of the superfinishing tool

$193 \mathrm{~m} \cdot \mathrm{min}^{-1}$

$9 \mathrm{~mm}$

$15 \mu \mathrm{m}$
The same parameters were entered into the simulation program. Among the input parameters of the simulation, there are also those that cannot be realistically determined. Therefore, the values of such parameters were chosen based on experience:

- the phase shift of paths $75^{\circ}$

- the depth of cut per workpiece rotation

$0.6 \mu \mathrm{m}$

- the ratio of cutting grain volume to tool volume

$40 \%$

On the produced surface, the surface roughness was measured at ten places. Simulation yielded 375 cuts - surface roughness profiles. The selected surface roughness parameters were determined on each profile. Table 1 presents the measured (12 profiles, tactile Surtronic 3+, Taylor Hobson) and simulated (375 profiles) values of the surface roughness parameters. For each parameter, the smallest value (min.), the largest value (max.) and the average value (arithmetic mean) are given.

A graphical model of the superfinished surface was also created. The actual dimensions of the modelled area are $1.2 \mathrm{~mm} \times 0.75 \mathrm{~mm}$ (which represents 375 cuts with a distance of $0.002 \mathrm{~mm}$ from each other). The real superfinished surface of the bearing ring was captured using a CCD camera. Both surfaces (simulated and real) are shown in Figure 7.

Table 1. Measured and simulated surface roughness values $[\mu \mathrm{m}]$

\begin{tabular}{|c|c|c|c|c|c|c|}
\hline \multirow{2}{*}{ The parameter } & \multicolumn{3}{|c|}{ The real surface } & \multicolumn{3}{c|}{ The simulated surface } \\
\cline { 2 - 7 } & min. & max. & the arithmetic mean & min. & max. & the arithmetic mean \\
\hline$R a$ & 0.11 & 0.14 & 0.123 & 0.138 & 0.136 & 0.137 \\
\hline$R z$ & 0.90 & 1.40 & 1.150 & 0.980 & 0.936 & 0.956 \\
\hline$R q$ & 0.14 & 0.18 & 0.158 & 0.173 & 0.170 & 0.172 \\
\hline$R t$ & 1.00 & 1.50 & 1.210 & 1.210 & 1.030 & 1.128 \\
\hline
\end{tabular}

a)

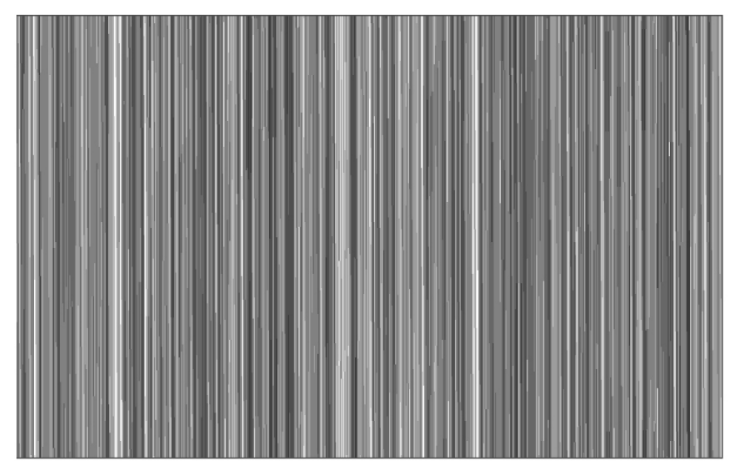

b)

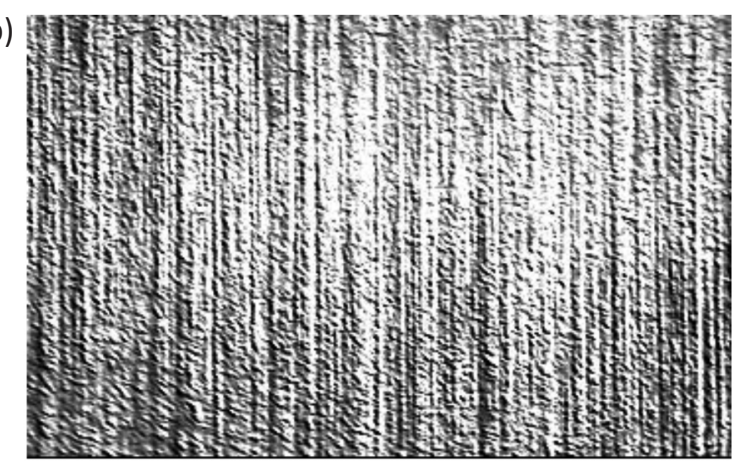

Fig. 7. Simulated (a) and real (b) superfinished surface (The surface size: $1.2 \times 0.75 \mathrm{~mm}$ ) 


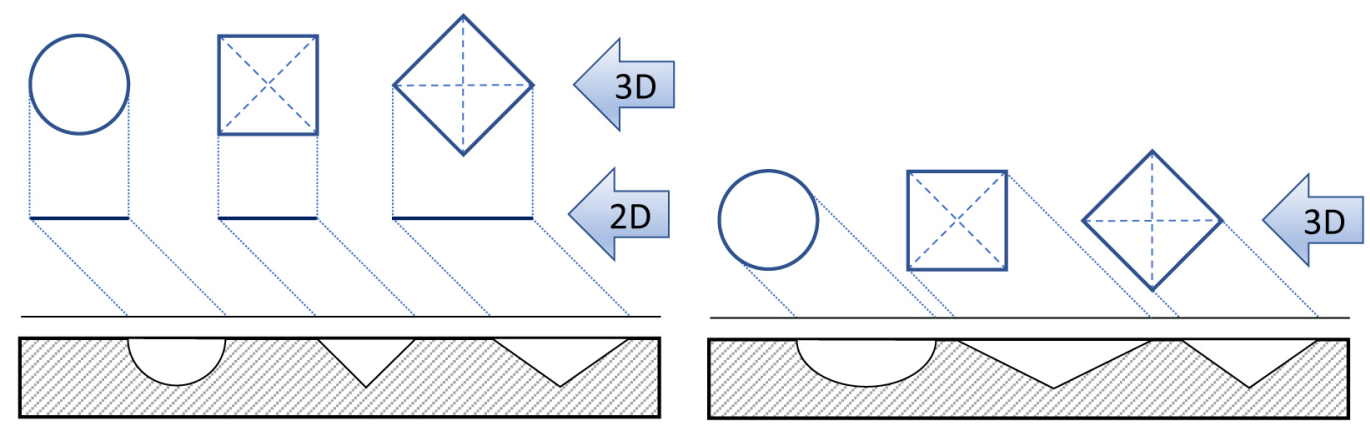

Fig. 8. The removal of material by $2 \mathrm{D}$ and $3 \mathrm{D}$ tool grains

\section{CONCLUSION}

The proposed simulation of material removal during superfinishing and thus the creation of a surface roughness profile and a graphical surface model is the result of the analysis of the cutting process. It can be included among the simulation research methods in the field of machining. It is another step on the way of using mathematical methods in science (research) and practice. The simulation needs to be further improved and refined.

A significant disadvantage of the simulation is that it was not considered with the three-dimensional (3D) grains of the tool. For simplicity, only two-dimensional (2D) grain profiles were used in the simulation. Therefore, even in the simulation numerical experiment, the crossing angle of the $\beta$ tracks was statistically insignificant. The difference between the simulation with $2 \mathrm{D}$ and $3 \mathrm{D}$ tool grains is presented in Figure 8.

Considering the tool wear and other random phenomena occurring during machining would also refine the simulation. The proposed method could be partially applied to other more kinematically complex methods of superfinishing (e.g. multi-oscillating) and other abrasive machining methods (e.g. honing).

\section{Acknowledgement}

This contribution was supported by research project VEGA 1/0019/20 "Accurate calculations, modelling and simulation of new surfaces based on physical causes of machined surfaces and additive technology surfaces in machinery and robotic machining conditions". This support is gratefully acknowledged.

\section{REFERENCES}

1. Grama, L., Gabor, M., Dattoma, V., Beno, J. Study of different process parameters on the surface roughness at superfinishing. Scientific Bulletin of the Petru Maior University of Tirgu Mures, Vol. 7 (XXIV), No. 1, 2010.

2. Brzhozovskii, B., Zakharov, O. More precise superfinishing by means of statistical modeling. Russian Engineering Research. 30, 2010, 1271-1275. https://doi.org/10.3103/S1068798X1012021X

3. Yamaguchi H. Superfinishing. In: Chatti S., Laperrière L., Reinhart G., Tolio (Eds.) CIRP Encyclopedia of Production Engineering. Springer, Berlin-Heidelberg 2019. https://doi.org/10.1007/978-3-64235950-76436-3

4. Zakharov, O.V., Datskovskaya, E.A. Setup of centerless superfinishing machine tools. Russian Engineering Research, 30(12), 2010, 1263-1267, https://doi.org/ 10.3103/S1068798X10120191

5. Zakharov, O.V. Principles for the adjustment of centerless superfinishing machines. Russian Engineering Research, 31(5), 2011, 465-468, https:// doi.org/10.3103/S1068798X11050261

6. Pawlus, P., Reizer, R., Wieczorowski, M. A review of methods of random surface topography modeling. Tribology International, 152, 2020.

7. Lepadatescu, B., Popa, L., Buzatu, C. Studies and researches regarding a mathematical model of superfinishing manufacturing process. Mechanical engineering; Recent advances in mechanical engineering and automatic control, 2012, 151-157.

8. Buzatu, C., Fota, A., Lepadatescu, B., Duicu, S. Modelling by finite element of the part-tool flexible technological system deformations at superfinishing process. In: Proceedings of the 8th WSEAS International Conference on Artificial Intelligence, Knowledge Engineering and Data Bases, 2009, 139-142.

9. Jiang, Q., Ge, Z. Simulation on topography of superfinished roller surfaces. Sc. China Ser. B-Chem. 45, 2002, 122-126. https://doi.org/10.1360/02yb9017 
10. Patir, N. A numerical model for random generation of rough surfaces. Wear, 45, 1977, 263-77. https:// doi.org/10.1016/0043-1648(78)90157-6

11. Liao, D., Shao, W., Tang, J., Li, J. An improved rough surface modeling method based on linear transformation technique. Tribol Int., 119, 2018, 786-94. https://doi.org/ 10.1016/j.triboint.2017.12.008

12. You, SJ, Ehmann, KF. Computer synthesis of threedimensional surfaces. Wear, 145, 1991, 29-42. https://doi.org/10.1016/0043-1648(91)90237-O.

13. Neagu-Ventzel, S., Cioc, S., Marinescu, I. A wear model and simulation of superfinishing process: analysis for the superfinishing of bearing rings, Wear, 26(9-10), 2006, 1061-1069.

14. Arrazola, P.: Modeling in cutting. CIRP Encyclopedia of Production Engineering, 2014, 1-7, https:// doi.org/10.1007/978-3-642-35950-7_16800-1.
15. Pawlus, P., Reizer, R., Wieczorowski, M., Krolczyk, G. Material ratio curve as information on the state of surface topography - A review. Precision Engineering, 65, 2020. https://doi.org/ 10.1016/j. precisioneng.2020.05.008.

16. Buzatu, C., Balacescu, A. Surface roughness modelling at ball bearing rings superfinishing on the cutting fluid and process parameters influence. In: Proc. of the International Conference on Economic Engineering and Manufacturing Systems, Braşov, 25-26 Oct. 2007, 8, 3a(21a).

17. Lepadatescu, B., Yordanova, S. Neuro-fuzzy logic based modelling for optimisation in superfinishing process. Advances in Automatic Control, Modelling \& Simulation. http://www. wseas.us/e-library/conferences/2013/Brasov/ ACMOS/ACMOS-06.pdf 